\title{
Gluteoplastía de aumento con implantes de silicona con técnica XYZ. Serie de casos
}

\author{
Stefan Danilla E. ${ }^{1,2}$, Diego Quispe V. ${ }^{1}$, Cristian Erazo C. ${ }^{1}$, Patricio Andrades C. ${ }^{1}$, \\ Rolando Schulz R. ${ }^{1}$, Claudia Albornoz G. ${ }^{1}$ y Sergio Sepúlveda P. ${ }^{1,2}$
}

\begin{abstract}
Augmentation gluteoplasty with silicone implants with XYZ technique
Objective: Describing the clinical features, demographics features, outcomes and complications from a serie of patients underwent buttock augmentation with silicone implants with XYZ technique. Material and Methods: Retrospective case series of patients underwent buttock augmentation with intramuscular technique. It Is described the surgical technique, demographics data, comorbidities and post operative complications. Results: Since December 2014 to June 2017 were operated 19 patients. The most frecuent complication was wound dehiscence, it was present in 5 patients. One patient had a postoperative hematoma. Two patients presented seroma. One patient presented implant rotation. One patient left controls after removal the suture in the sixth week. None of patients needed revisional procedures. None of patients presented wound infection. None of patients presented extrusión or implant removal. Discussion: The $\mathrm{XYZ}$ technique is a efective method to aesthetic and reconstructive treatment of buttock area, especially in patientes without a suficient donor area to fat graft. The complications described are accord with literatura, that is why buttock augmentation with intramuscular silicone implant is recomended as a reproducible and safe technique.

Key words: buttock; esthetic; gluteoplasty; implants; silicone.
\end{abstract}

\section{Resumen}

Objetivo: Describir las características clínicas, demográficas, resultados y complicaciones de una serie de pacientes operados de aumento de glúteo con implantes de silicona por medio de la técnica XYZ. Materiales y Método: Serie de casos retrospectiva de pacientes sometidos a gluteoplastía de aumento con implantes de silicona por medio de técnica intramuscular. Se describe la técnica quirúrgica, datos demográficos, antecedentes médico quirúrgicos y complicaciones posoperatorias. Resultados: Entre diciembre de 2014 y junio de 2017 se operaron 19 pacientes. La complicación más frecuente fue dehiscencia de herida operatoria, presentándose en 5 pacientes. Un paciente tuvo hematoma posoperatorio. Dos pacientes tuvieron seroma posoperatorio. Una paciente presentó rotación de un implante. Una paciente se perdió de seguimiento luego del control de retiro de puntos a la $6^{\mathrm{a}}$ semana. Una paciente necesitó reinternación para manejo del dolor. Ningún paciente necesitó reoperación. Ningún paciente presentó infección de herida operatoria. Ningún paciente presentó extrusión ni pérdida de implantes. Discusión: La técnica XYZ es un método efectivo para el tratamiento estético y reconstructivo de la región glútea especialmente en pacientes que no presentan zona dadora suficiente para injerto graso. Las complicaciones descritas son acordes a la literatura, por lo que se recomienda la técnica de gluteoplastía de aumento con prótesis de silicona intramuscular como una técnica reproducible y segura.

Palabras clave: glúteo; estética; gluteoplastía; implantes; silicona.

\section{Introducción}

A lo largo de la historia, a través de las culturas y el tiempo, la cadera y los glúteos han perdurado como un elemento clave del cuerpo y belleza femenina. El arte pictórico, escultórico y otras formas de bellas artes, han representado la figura femenina estética ideal. En los últimos años ha habido un aumento dramático en el número de pacientes que buscan perfeccionar sus figuras a través de la cirugía estética, con un énfasis notable en la región glútea, la cual ha aumentado $18 \%$ entre el 2015 y el $2016^{1,2}$.
Departamento de Cirugía Hospital Clínico Universidad de Chile.

${ }^{2}$ Clínica Alemana de Santiago. Santiago, Chile.

Conflictos de interés: No hay.

Recibido el 21 de enero de 2018 y aceptado para publicación el 19 de abril de 2019.

Correspondencia a:

Dr. Stefan Danilla E. drstefandanilla@gmail.com 
Entre las técnicas de aumento de contorno glúteo, se encuentra la gluteoplastía de aumento, que se puede lograr mediante implantes ${ }^{3}$ o lipoinyección ${ }^{4,5}$; y el lifting o levantamiento glúteo con aumento autólogo en el contexto de una abdominoplastía circunferencial ${ }^{6,7}$.

El primer procedimiento de aumento glúteo se remonta a 1969, en que Bartels y cols describen una reconstrucción glútea unilateral utilizando un implante mamario subcutáneo ${ }^{8}$. Algunos años más tarde, en 1973, Cocke y Rickerson presentan el primer aumento exitoso con fines cosméticos, aplicando un implante redondo específico para glúteos, con mayor proyección que el implante mamario9. En 1975 se introduce el término "platipigia" por Douglas, que se refiere al déficit de tejido en glúteos o glúteos planos ${ }^{10}$. Si bien fue en Estados Unidos donde se inició el desarrollo de este tipo de cirugías, fue en Latinoamérica con González-Ulloa, en 1991, que se popularizó y perfeccionó la técnica de aumento glúteo como procedimiento estético, con implantes subcutáneos a través de incisiones en los pliegues infraglúteos la cual fue rápidamente abandonada por sus resultados poco naturales y alta tasa de complicaciones ${ }^{11}$. Se han descrito varias técnicas, cuya principal diferencia está dada por la ubicación de los implantes. En 1984, Robles y cols detallan la colocación en el plano submuscular, bajo el músculo glúteo mayor a través de una incisión única a nivel del sacro ${ }^{12}$. Unos años más tarde De la Peña describe el posicionamiento en el plano subfascial $^{13}$, sin embargo, la fascia glútea no es constante entre individuos y sus resultados también son poco naturales siendo evidente el borde del implante en muchos pacientes por lo cual al igual que la técnica subcutánea cada vez se utiliza menos. Vergara y Marcos, en 1996, describieron el posicionamiento intramuscular de los implantes ${ }^{14}$. Sin embargo, en el 2004, Raúl González estandariza y sistematiza la técnica detallando los niveles y límites de la disección intramuscular ${ }^{15}$, lo que facilitó la curva de aprendizaje y masificó el procedimiento debido a sus resultados seguros, eficaces y reproducibles.

\section{Objetivo}

El objetivo del presente trabajo es describir los resultados y complicaciones obtenidas por el autor principal con la técnica XYZ de gluteoplastía de aumento intramuscular.

\section{Materiales y Método}

\section{Pacientes}

Se incluyeron todos los pacientes sometidos a gluteoplastía de aumento con implantes y se revi- saron los registros de la atención médica. El perfil de pacientes son mujeres y hombres con platipigia, delgados, lo que hace que la lipoinyección de grasa no sea una opción adecuada en estos pacientes o que su motivo principal de consulta es el aumento de volumen y proyección de la región glútea.

\section{Técnica quirúrgica}

Se utilizó la técnica XYZ de Gonzales ${ }^{15}$. Los pacientes fueron operados bajo anestesia general en decúbito prono, la preparación de piel se realizó con clorhexidina incolora acuosa o povidona yodada. La marcación preoperatoria identifica el sacro, crestas ilíacas, espina iliaca posterosuperior, coxis, trocánter mayor del fémur y masa del músculo glúteo mayor.

Una vez delimitados los puntos anatómicos de reparo se diseña la incisión en losanjo en el pliegue interglúteo con el fin conservar una isla cutánea que contenga el ligamento sacrocutáneo y de esa manera obtener abordajes independientes para cada glúteo, separados por $5 \mathrm{~mm}$, la incisión tiene como límite superior el borde superior del pliegue interglúteo y se prolonga hacia caudal $7 \mathrm{~cm}$. En relación a la incisión se diseña un bolsillo de forma de corazón invertido el cual tiene como eje las fibras del músculo glúteo mayor y una longitud de $7 \mathrm{~cm}$ en cada lado (Figura 1).

Una vez realizado el diseño se delimita el punto $\mathrm{X}$ que corresponde al punto donde se inserta el músculo glúteo mayor (MGM) en el meridiano del diseño del bolsillo subcutáneo, el punto Y que corresponde al límite superior del MGM sobre el meridiano del ala ilíaca y el punto $\mathrm{Z}$ que correspon-

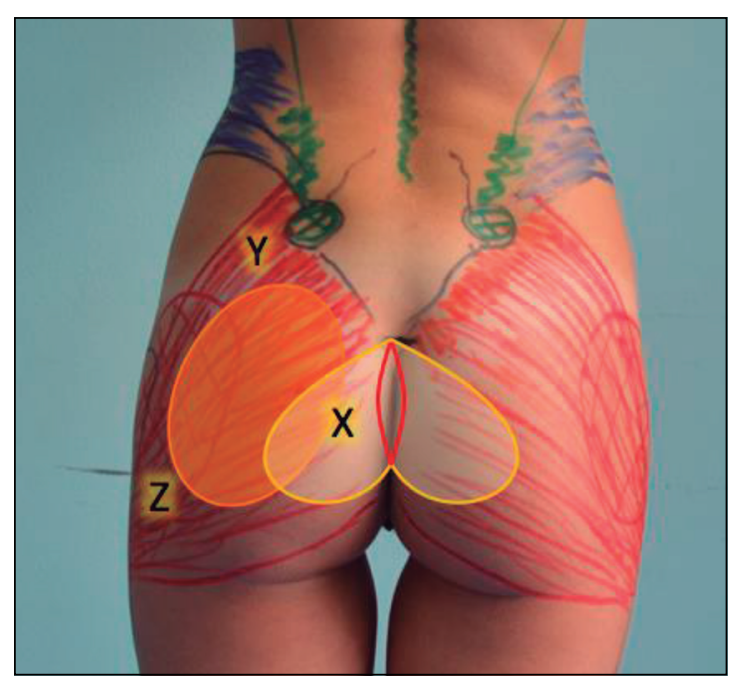

Figura 1. Marcación preoperatoria. Reparos anatómicos y planificación de abordaje quirúrgico mediante losanjo interglúteo y bolsillo en forma de corazón invertido. 
de al límite lateral del MGM en el trocánter mayor del fémur (Figura 1).

Una vez finalizada la marcación, se infiltra el espacio del bolsillo subcutáneo con $60 \mathrm{~mL}$ de solución de vasocontricción (epinefrina 1:1.000.000) y el área de la cintura con técnica húmeda o superhúmeda para liposucción. La liposucción de la cintura se considera parte de la técnica, ya que cumple con 2 objetivos: 1) quitar volumen en el área de los flancos y la cintura lo que contribuirá a un mejor resultado y 2) recolectar grasa para lipoinyección selectiva de áreas específicas como la depresión isquiática, área supratrocantérica y suavizar el reborde del implante en pacientes muy delgados.

Luego de la liposucción, se inicia la gluteoplastía de aumento propiamente tal. Se escinde la piel en $90^{\circ}$ solo hasta pasar la dermis y luego se angula el bisturí hacia lateral en $60^{\circ}$ para abordar el músculo preservando el ligamento sacrocutáneo que conforma el pliegue interglúteo. Una vez alcanzado el músculo la disección se continúa en forma suprafascial, lo que posteriormente ayudará al cierre. Una vez completada la disección subcutánea, en el punto X se divulsionan las fibras del músculo hasta una profundidad mínima de 2 centímetros, luego, se introduce el decolador entre las fibras en dirección cefálica hacia el punto Y, teniendo cuidado de no superficializarse ni de traspasar el MGM. Una vez alcanzado el punto Y, el decolador se rota hacia caudal hasta alcanzar el punto $\mathrm{Z}$, lo que completa la bipartición del MGM. La disección se completa con decoladores hasta crear un bolsillo suficiente para que quepa el implante deseado.

Es importante recalcar que, a diferencia de la mamoplastía de aumento, en que la paciente elige el tamaño de sus mamas y virtualmente se puede poner casi cualquier volumen, en el aumento glúteo sólo se debe introducir un implante que quepa cómodamente dentro del MGM y permita cerrar sin tensión el bolsillo intramuscular. El forzar la técnica traerá resultados inestéticos y poco naturales, siendo propenso a complicaciones a corto y largo plazo.

Una vez terminada la disección del bolsillo se realiza hemostasia con cauterio bipolar, a pesar de lo que pudiera pensarse, el MGM en general sangra poco luego de su bipartición. Finalizada la hemostasia y prueba de volúmenes, se introduce el implante definitivo y se realiza cierre por planos con vicryl 2.0 al músculo, puntos de capitonaje a la disección subcutánea. Se reseca la epidermis de la piel del losanjo y se reconstruye el ligamento interglúteo con vicryl

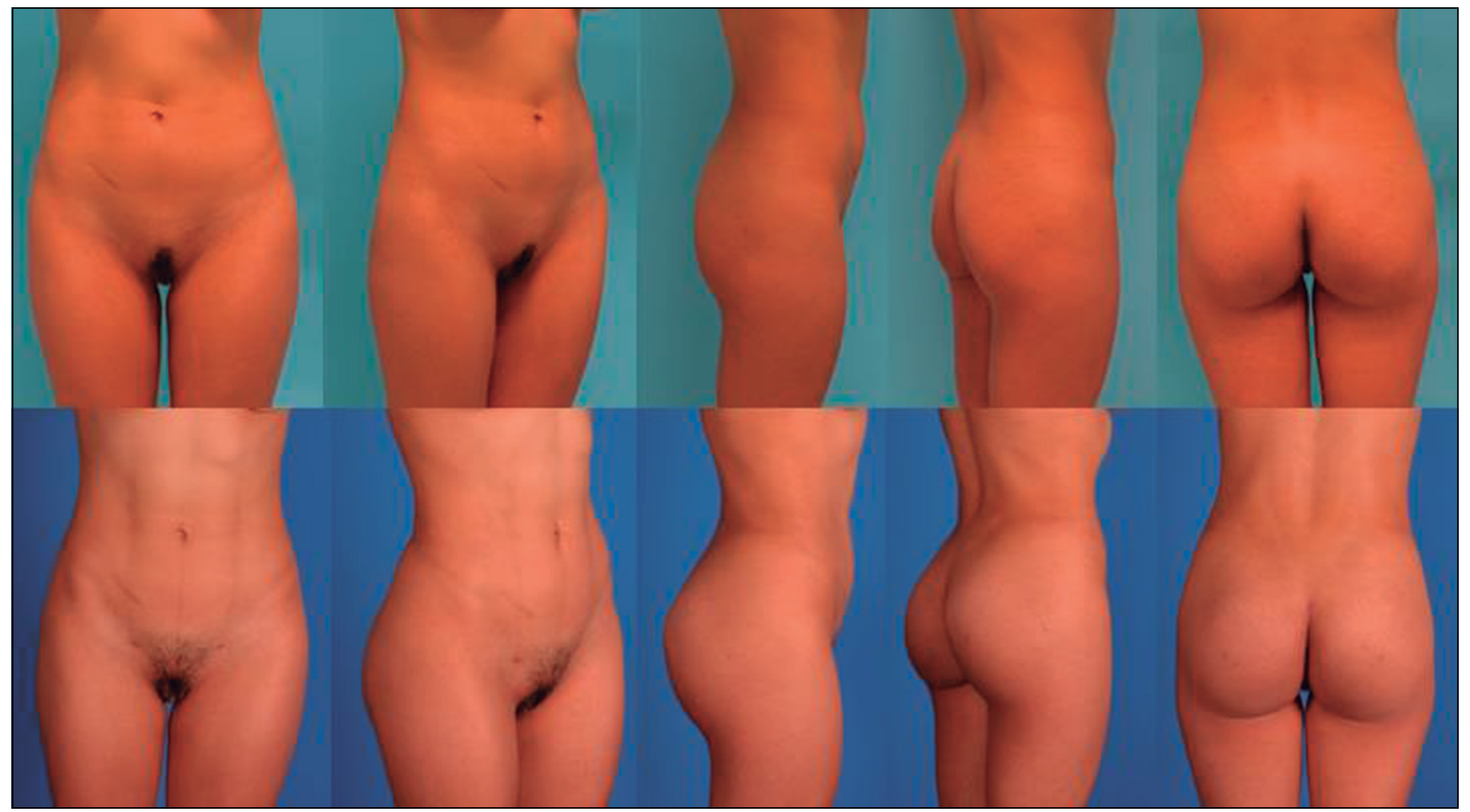

Figura 2. Fotografías pre y posoperatorias de paciente sometida a gluteoplastía de aumento con prótesis de silicona perfil alto $360 \mathrm{~mL}$ y lipoescultura de alta definición. 


\section{ARTÍ́CULO ORIGINAL}

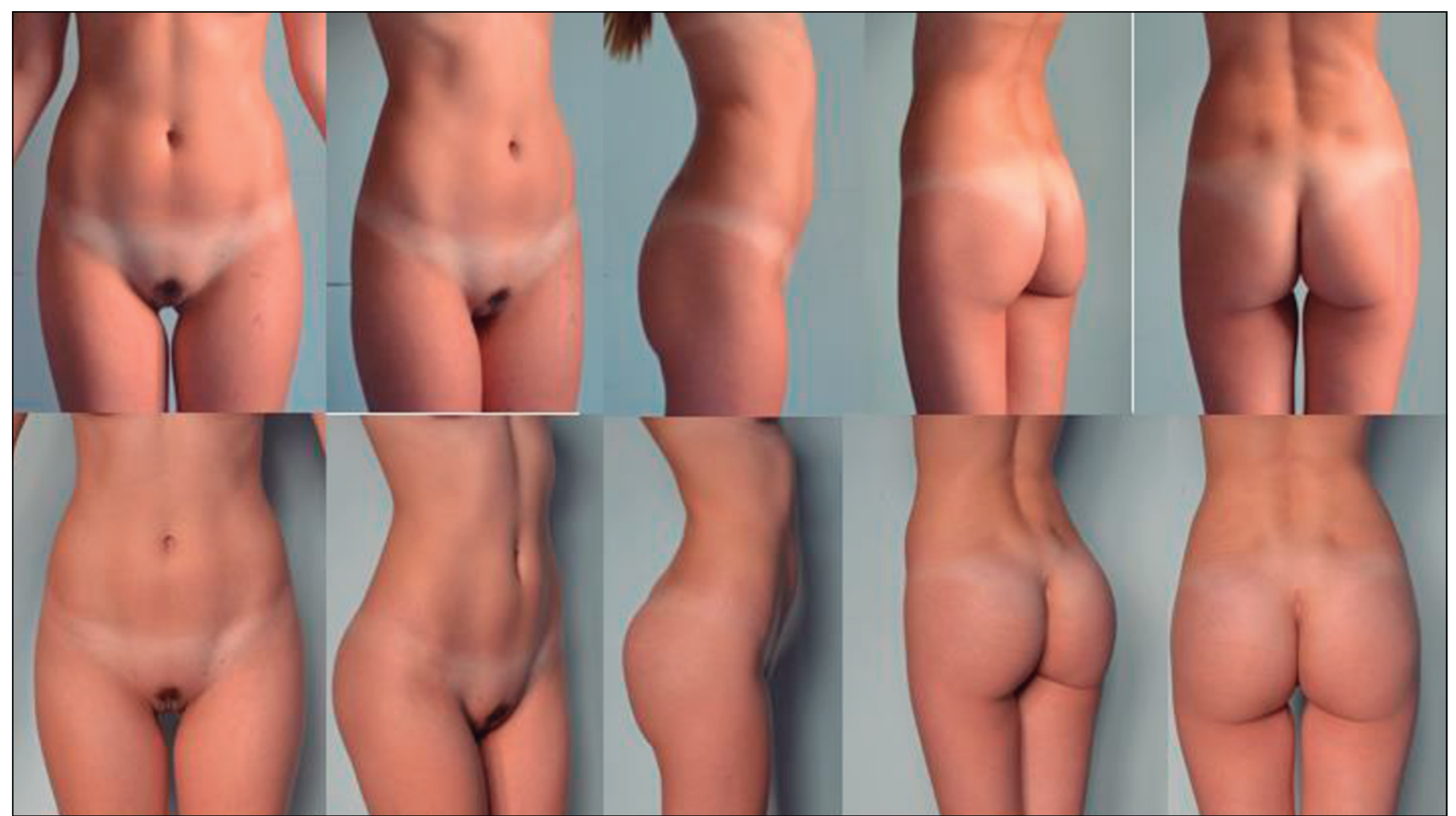

Figura 3. Fotografías pre y posoperatorias de paciente sometida a gluteoplastía de aumento con prótesis de silicona perfil alto de $305 \mathrm{~mL}$ y lipoescultura de alta definición.

Figura 4. Detalle de resultado alejado de paciente con dehiscencia de sutura con cierre por segunda intención.
2.0 para, posteriormente, cerrar la dermis con vicryl 4.0 dérmico profundo y piel con prolene 5.0 corrido.

Finalmente, si es necesario se realiza lipoinyección subcutánea en el área supratrocantérica y para suavizar el borde del implante si fuera evidente. En la presente serie no se utilizaron drenajes (Figuras 2 y 3 .

\section{Cuidados posoperatorios}

En el posoperatorio usualmente se indican analgésicos en dosis plenas asociando acetaminofeno,

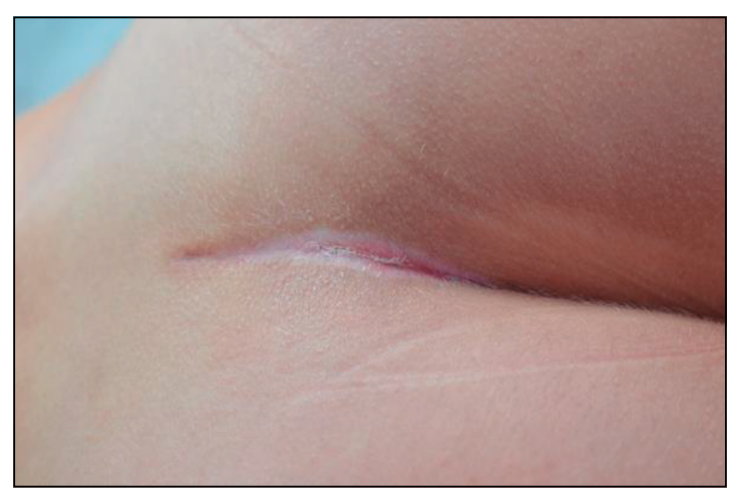

AINEs y tramadol ya que la cirugía es dolorosa durante los 5 primeros días. Además, se asocian antibióticos orales (cefadroxilo + metronidazol) y aseo meticuloso de la herida con duchoterapia al menos 3 veces al día y luego de cada deposición con abundante agua, jabón y ungüento antibiótico (mupirocina) luego del aseo.

No se realiza preparación de colon ni se restringe la posición del paciente. El paciente puede sentarse y acostarse en posición supina desde el día mismo de la cirugía. Tampoco se indica en forma rutinaria masajes de drenaje linfático ni ultrasonido.

Los pacientes habitualmente permanecen hospitalizados durante 1 día. Se estimula la deambulación precoz. El primer control se realiza a la semana posoperatoria para monitorizar la aparición de complicaciones y los puntos de prolene se retiran a la $3^{\mathrm{a}}$ o $4^{\mathrm{a}}$ semana para garantizar la atingencia a los controles por parte del paciente.

Como cuidado posoperatorio tardío, no se recomienda actividad física de alta intensidad en la región glútea a permanencia tales como sentadillas, estocadas, leg press, maratón o cualquier ejercicio localizado de glúteo. 


\section{Resultados}

\section{Características generales de los pacientes}

Entre diciembre de 2014 y junio de 2017 se han operado un total de 19 pacientes, 18 mujeres y 1 hombre. En todos los pacientes salvo 1 de sexo femenino se realizó la técnica descrita, la excepción corresponde a una mujer con atrofia casi total del MGM izquierdo secundario a una secuela de un accidente cerebrovascular, en el cual se realizó un bolsillo subfascial con el consentimiento previo de la paciente. De la serie 3 pacientes presentaban comorbilidades importantes, 1 paciente era portador de virus de inmunodeficiencia humana (VIH) en control y tratamiento, 1 tenía antecedentes de un infarto cerebral secundario a la ruptura de un aneurisma cerebral tratado y 1 tenía lupus eritematoso sistémico (LES) en tratamiento. Las características generales de los pacientes se detallan en la Tabla 1.

En todos los pacientes la cirugía se complementó con liposucción de la cintura. Como cirugías combinadas, además, se realizaron mastopexia en 2 pacientes, lipoescultura de alta definición en 3 pacientes y aumento mamario en 1 paciente (Tabla 2).

En todos los pacientes se utilizaron implantes de silicona ovales (anatómicos), el volumen más utilizado fue el de $305 \mathrm{~mL}$ seguido por $240 \mathrm{~mL}$ y $365 \mathrm{~mL}$. En 1 paciente se utilizaron implantes asimétricos, correspondiente a la paciente con secuela de accidente cerebrovascular descrita previamente, en todos los demás se empleó el mismo volumen de implante para el glúteo derecho e izquierdo. Tan solo 1 paciente utilizo implantes de perfil bajo, el resto fue de perfil alto (Tabla 3 ).

\section{Complicaciones}

La complicación más frecuente fue la dehiscencia del tercio inferior (cercana al ano) en 5 pacientes. En todos se resolvió en forma espontánea con cierre por segunda intención sin secuelas cicatrizales importantes (Figura 4).

Un paciente tuvo hematoma posoperatorio el cual se drenó en forma espontánea y no fue necesario re intervenir a la paciente ni presentó complicaciones profundas asociadas al implante.

Dos pacientes tuvieron seroma, uno fue prolongado por lo que requirió múltiples punciones evacuadoras, este paciente correspondió al implante subfascial en la paciente con atrofia del MGM secuela de un accidente cerebro vascular, el otro paciente se corresponde con la paciente que presentaba antecedentes de lupus eritematoso sistémico en tratamiento.

Tabla 1. Datos demográficos de pacientes sometidas a gluteoplastía con implantes

\begin{tabular}{|ll|}
\hline Datos demográficos de los pacientes & \\
\hline Edad (promedio +/- DE, Rango) & 35 (DE 8,2) 21-50 \\
\hline Peso (promedio +/- DE, Rango) & $56(\mathrm{DE} 5,8)$ 47-66 \\
\hline Talla (promedio +/- DE, Rango) & 1,6 (DE 0,07) 1,5-1,7 \\
\hline IMC (promedio +/- DE, Rango) & 22 (DE 1,5) 18-24 \\
\hline Tiempo operatorio (promedio +/- DS, Rango) & 235 min. (DE 119) 135-570 \\
\hline Comorbilidades & 3 pacientes \\
$\quad-1$ LES & \\
$\quad-1$ VIH & \\
\hline Cirugías estéticas previas & 5 pacientes \\
Cirugías no estéticas previas & 9 pacientes \\
\hline Tabaco & 2 pacientes \\
\hline
\end{tabular}

Tabla 2. Procedimientos complementarios asociados a gluteoplastía con implantes

Procedimientos complementarios a la gluteoplastía $\mathbf{N}^{\circ}$ de pacientes

Lipoaspiración convencional

Lipoaspiración de alta definición

3

Mastopexia

Aumento mamario

Tabla 3. Tipo de implantes utilizados en pacientes sometidas a gluteoplastía con implantes

\begin{tabular}{|c|c|c|c|}
\hline \multirow[t]{2}{*}{ Tipo de implantes } & \multirow[t]{2}{*}{ Número de pacientes } & \multicolumn{2}{|c|}{ Media volumen de implantes } \\
\hline & & derecho & izquierdo \\
\hline Oval perfil alto & 18 & 270 & 269 \\
\hline
\end{tabular}

Tabla 4. Complicaciones posoperatorias gluteoplastía con implantes

\section{Complicaciones posoperatorias}

Dehiscencia

Seroma

Hematoma

Rotación implante

Dolor agudo

\section{$\mathbf{N}^{0}$ de pacientes}

5

2

1

1 
Una paciente presentó rotación de un implante, se trató de una paciente con una secuela cicatrizal antigua del MGM producto de un absceso intramuscular secundario a una inyección intramuscular de larga data, identificando durante la cirugía una cicatriz intramuscular. Una paciente se perdió del seguimiento luego del control de retiro de puntos a la $6^{\mathrm{a}}$ semana. Una paciente necesitó reinternación para manejo del dolor (Tabla 4).

Ningún paciente necesitó reoperación, ningún paciente presentó infección de herida operatoria ni extrusión ni pérdida de implantes. Hubo una alta satisfacción con la cirugía excepto en 1 paciente.

\section{Discusión}

El aumento de glúteos con implantes esta especialmente indicado en pacientes con una ptosis moderada a severa o asimetría glútea sin suficiente volumen de grasa que permita una lipotransferencia, las complicaciones más comúnmente reportadas son dehiscencia de herida $(9,6 \%)$, seroma $(4,6 \%)$, infección $(1,9 \%)$, revisión de implantes $(1,7 \%) \mathrm{y}$ parestesia ciática transitoria $(1,1 \%)^{16}$. La lipoinyección es una excelente técnica debido a que permite aumentar y moldear los glúteos en forma dirigida con menor tasa de complicaciones, sin embargo, está indicada en pacientes con un adecuado sitio donante y que no requieren de una proyección muy importante, las complicaciones más frecuentes del injerto graso son seroma $(3,5 \%)$, Falta de corrección $(2,1 \%)$, Infección $(1,9 \%)$ y asimetría $(0,6 \%)^{16}$.

En la presente serie no se presentaron complicaciones mayores, solo una paciente tuvo un resultado estético insatisfactorio producto de la rotación de un implante, este paciente presentaba antecedentes de un absceso intramuscular secundario a una inyección intramuscular de larga data por lo que es probable que esté relacionado con la cicatriz intramuscular que se diagnosticó en forma intraoperatoria. Desafortunadamente no existen estudios que realicen un seguimiento a largo plazo de un número significativo de pacientes con gluteoplastía de aumento ${ }^{17}$. La complicación tardía más importante es la ptosis del implante y la rotación del implante debido a expansión del bolsillo, si bien la incidencia no ha sido reportada hay consenso de que existe, pero no es reportado ${ }^{17}$. La literatura sugiere que es mas probable en el plano subfascial que en el plano intramuscular o submuscular ${ }^{17}$. Un estudio que evalúa la posición de los implantes con tomografía axial computarizada reveló que todos los implantes ovales intramusculares que estudia- ron modificaban su posición vertical a dirección oblicua en sentido de las fibras del músculo a los 3 meses, sin embargo, no provoca asimetría debido a que el cambio de posición fue simétrico en ambas prótesis; en ese mismo estudio se evidenció rotación con la base del implante hacia superficial tanto en implantes redondos como ovales ${ }^{18}$. La rotación de un implante glúteo parece ser atribuido a la excesiva disección y, por lo tanto, un inadecuado tamaño del bolsillo intramuscular que se confecciona ${ }^{18}$. Por lo que la debilidad de la pared muscular de la paciente que presentó rotación del implante, en este caso producto de una secuela cicatrizal, puede haber determinado la rotación.

Se presentaron dos casos de seroma los cuales se corresponden con el paciente que presentaba antecedentes de un infarto cerebral y con el paciente que tenía antecedentes de lupus eritematoso sistémico en tratamiento lo que podría haber condicionado la aparición de estos. La tasa de seroma en la literatura varía de $3,7 \%$ a $19 \%$, solo en algunos estudios utilizaron drenaje, los estudios que menos utilizaron drenaje fueron aquellos con técnica primaria intramuscular o submuscular ${ }^{17}$. Existe una gran variedad en los estudios con respecto al tipo de implante, tamaño de implante, localización del implante, uso de drenaje y textura del implante lo que no permite establecer recomendaciones confiables para su prevención ${ }^{17}$. Senderoff describe una reducción de la tasa de seroma de $9,09 \%$ a $2 \%$ con el uso de puntos de capitonaje en prótesis subfasciales ${ }^{19}$. En esta serie de casos el cirujano no ocupa drenaje y realiza puntos de capitonaje durante el cierre operatorio.

Contractura capsular es una rara complicación en el aumento de glúteos con implantes, Sinno y cols $^{16}$ realizaron una revisión de todo lo publicado hasta abril de 2015 reportando una tasa de 0,5\% (1 paciente que presentó contractura después de 2 años del implante).

En opinión del autor, la principal dificultad de la técnica descrita por el Dr. González es que la descripción del procedimiento fue realizada en su mayoría en pacientes brasileñas, existiendo diferencias anatómicas importantes entre éstas y las chilenas $^{20}$. Las pacientes chilenas tienen un espesor del MGM promedio de $3,8 \mathrm{~cm}$, lo cual es menor al de las pacientes brasileñas que en promedio tienen 4 a $6 \mathrm{~cm}$ de espesor ${ }^{20}$. Por lo tanto, se debe tener especial cuidado al realizar el bolsillo intramuscular para el implante en la población chilena, pues existe evidencia que sugiere menor espesor del músculo glúteo y, si bien Gonzales ${ }^{15}$ en su artículo refiere que se debe asegurar una cobertura del implante con MGM superficial de 2,5 cm, en población chilena 
se podría utilizar la recomendación de otros autores que refieren que con $2 \mathrm{~cm}$ es suficiente ${ }^{21}$. Otra dificultad técnica es la disección del músculo en su porción inferior para el adecuado posicionamiento del implante en situación anatómica ideal para evitar el desplazamiento a cefálico y una apariencia poco natural.

Es necesario el seguimiento a más largo plazo para poder determinar el resultado alejado de la técnica. Ha sido reportado atrofia de la porción superficial del MGM luego de su bipartición, la cual no afecta la función del músculo a largo plazo ${ }^{22}$.

En la experiencia del autor, la satisfacción de las pacientes ha sido alta.

En Chile ha existido un lento desarrollo de este tipo de cirugía, creemos que se debe a que son pocos los cirujanos que están familiarizados con la técnica quirúrgica y por muchos años el procedimiento estuvo estigmatizado por altas tasas de complicaciones, lo que desmotivó su uso. En la actualidad, existe cierto consenso y evidencia que el plano intramus- cular para gluteoplastía es un procedimiento seguro con tasas de complicaciones comparables con otros procedimientos estéticos ${ }^{23}$.

En suma, los autores consideran la técnica XYZ como un método efectivo y seguro para el embellecimiento de la región glútea, puesto que la tasa de complicaciones presentada es baja y las complicaciones son leves; creemos que el beneficio justifica sus riesgos.

\section{Responsabilidades éticas}

Protección de personas y animales. Los autores declaran que para esta investigación no se han realizado experimentos en seres humanos ni en animales.

Confidencialidad de los datos. Los autores declaran que en este artículo no aparecen datos de pacientes.

Derecho a la privacidad y consentimiento informado. Los autores declaran que en este artículo no aparecen datos de pacientes.

\section{Referencias}

1. ISAPS - ISAPS Global Statistics [Internet]. [cited $2016 \mathrm{Jul}$ 7]. Available from: http://www.isaps.org/news/isapsglobal-statistics.

2. American Society of Plastic Surgeons. 2016 Plastic Surgery Statistics Report [Internet]. ASPS Public Relations; Available from: https://www. plasticsurgery.org/documents/News/ Statistics/2016/plastic-surgery-statisticsfull-report-2016.pdf

3. Vergara R, Amezcua H. Intramuscular Gluteal Implants: 15 Years' Experience, Aesthet Surg J. 2003;23:86-91.

4. Cárdenas-Camarena L, Lacouture AM, Tobar-Losada A, Combined gluteoplasty: liposuction and lipoinjection. Plast Reconstr Surg. 1999;104:1524-31.

5. Mendieta C. Gluteal reshaping. Aesthetic Surgery Journal. 2007;27:641-55.

6. Centeno RF. Autologous Gluteal Augmentation With Circumferential Body Lift in the Massive Weight Loss and Aesthetic Patient. Clinics in Plastic Surgery. 2006;33:479-96.

7. Richter DF, Stoff A. Circumferential Body Contouring: The Lower Body Lift. Clinics in Plastic Surgery. 2014;41:77588.

8. Bartels RJ, O'Malley JE, Douglas WM. An unusual use of the cronin breast prosthesis: Case Report, Plastic and Reconstructive Surgery. 1969;44:500.

9. Cocke WM, Ricketson G. Gluteal augmentation. Plast Reconstr Surg. 1973;52:93.

10. Dougles WM, Bartels RJ, Baker JL. An experience in aesthetic buttock augmentation. Clin Plast Surg 1975;2:471.

11. González-Ulloa M. Gluteoplasty: a ten-year report. Aesthetic Plast Surg. 1991;15:85-91.

12. Robles JM, Tagliapietra JC, Grandi MA Gluteoplastía de aumento: implante submuscular. Cir Plast Ibero Latinoam. 1984;10:123-9.

13. de la Peña JA, Rubio OV, Cano JP, Cedillo MC, Garcés MT. Subfascial Gluteal Augmentation. Clinics in Plastic Surgery 2006;33:405-22.

14. Vergara R, Marcos M. Intramuscular gluteal implants. Aesthetic Plast Surg. 1996;20:259-62.

15. González R. Augmentation Gluteoplasty: The XYZ Method. Aesth Plast Surg. 2004;28:417-25.

16. Sinno S, Chang JD, Brownstone ND, Saadeh PB, Wall S. Determining the Safety and Efficacy of Gluteal Augmentation: A Systematic Review of Outcomes and Complications. Plastic and Reconstructive Surgery. 2016;137:1151-6.

17. Shah B. Complications in Gluteal Augmentation. Clinics in Plastic Surgery.
2018; 45:179-86.

18. Serra F, Aboudib JH, Marques RG. Intramuscular Technique for Gluteal Augmentation: Determination and Quantification of Muscle Atrophy and Implant Position by Computed Tomographic Scan. Plastic and Reconstructive Surgery. 2013;131:253e-259e.

19. Senderoff DM. Buttock Augmentation With Solid Silicone Implants. Aesthet Surg J. 2011;31:320-327.

20. Troncoso E, Ríos M, Carrasco J, Cuevas P, Cisternas JP, Erazo C, et al. Espesor normal del músculo glúteo mayor en mujeres chilenas: Una guía para el aumento de glúteo con implantes. Revi Chil Cir. 2016;68:427-32.

21. Vergara R, Amezcua H. Intramuscular gluteal implants: 15 years' experience. Aesthet Surg J. 2003;23(2):86-91.

22. Serra F, Aboudib JH, Neto JI, Cossich VR, Rodrigues NC, de Oliveira KF, et al. Volumetric and Functional Evaluation of the Gluteus Maximus Muscle after Augmentation Gluteoplasty Using Silicone Implant. Plastic and Reconstructive Surgery 2015;135:533e-541e.

23. Flores-Lima G, Eppley BL, Dimas JR, Navarro DE. Surgical Pocket Location for Gluteal Implants: A Systematic Review. Aesth Plast Surg. 2013;37:240-5. 